\title{
Liquid-liquid phase transition: is it possible in Fe melts?
}

\author{
A.A. Mirzoev, A.N. Sobolev \\ South-Ural State University, Physical Department, 454080 Chelyabinsk, Russia
}

\begin{abstract}
In present work we present the results of ab initio molecular dynamics simulation of magnetic properties of liquid iron. This simulation has been performed using the computer package SIESTA for temperature range: $1833,1873,1923,2023 \mathrm{~K}$. Our focus is on possibility of magnetic liquid-liquid phase transition in iron melts.
\end{abstract}

\section{Introduction}

Last years considerable progress is observed in the research of liquid-liquid phase transitions. Existence of such transitions in net forming liquids $\left(\mathrm{SiO}_{2}, \mathrm{P}\right)$ is reliably established [1-2]. The theoretical background for liquid-liquid phase transitions is offered by Tanaka [3] on the basis of two-state model. According to this model two various types of local structure can compete in a liquid. One state is better packed (small specific volume) and it has a higher entropy, but at the expense of a higher energy. The other state is a low-energy one, but it has open structure (large specific volume), and due to some reason it has a low entropy. In case of liquid metals it is difficult to expect existence of strongly different local structures. Therefore existence of polymorphic transitions in metal melts is still debatable now. Due to the practical importance, the question about the possibility of structural transitions in liquid iron is especially interesting. It has been discussed for more than 40 years since the publication of Vertman and Samarin [4].

Available experimental data about Fe melts properties are inconsistent to each other. However some behaviour features of magnetic susceptibility and viscosity temperature dependence in the temperature range of 1580 to $1640^{\circ} \mathrm{C}$ are found out $[4,5]$. If the features specified really exist, the question arises, if there are several different types of structures which compete with each other.

Magnetism is known to be important in the phase stability, structure and elastic properties of iron. For example, the ground state of $\alpha-\mathrm{Fe}$ at ambient conditions would be mechanically unstable if it is not magnetic. Even much above the Curie temperature $T_{c}$ there are local magnetic moments in $\alpha-\mathrm{Fe}$ [6-10].

For this reason the main subject of our report is to investigate the magnetic properties of iron melts in wide range of temperatures. Our goal is to show that in case of liquid iron the competing structures are different in magnetic order- ing, but not in density of packing. We use ab initio molecular dynamic simulations computer package SIESTA [11] for modeling liquid iron at the temperatures of 1833 to $2023 \mathrm{~K}$.

\section{Computational details}

In this work we utilise iron pseudopotential which was proposed by Izquierdo et al. in [12]. We have used GGA in Perdew-Wang-Ernzerhof parameterisation [13] as an approximation for exchange-correlation potential. Non-linear corrections to the exchange-correlation terms proposed by Louie [14] are also explicitly stated in pseudopotential. We use the "standard" double- $\zeta$ plus polarization basis sets and MD time step of $1 \mathrm{fs}$, and reach total simulated time of order $1.5-2.0$ ps with a total of $N=256$ atoms. For all runs we employed spin polarization, reasoning that local magnetic moments exist even above the Curie point. These local moments have a significant influence on the shortrange order because ferromagnetic iron prefers a longer bond length than paramagnetic iron[15].

\section{Results}

In Fig. 1, the magnitude of the local magnetic moment of an atom (in units of $\mu_{B}$ ) of liquid iron is plotted against its Voronoi volume. The dependences of the magnetic moments for BCC and FCC iron crystals are shown by continuous lines. Evidently, the moment distribution for liquid iron is fairly near to the curve for FCC-Fe than for the same one for BCC-Fe. This indicates that liquid metal structure is quite similar to denser FCC-phase. We can point out that the presented curve is in a good qualitative accordance with the same results for amorphous $\mu-\mathrm{Fe}$ [16]. The presented distribution is broader therefore it is expected because local structure in liquid state is more variable than in amorphous. 


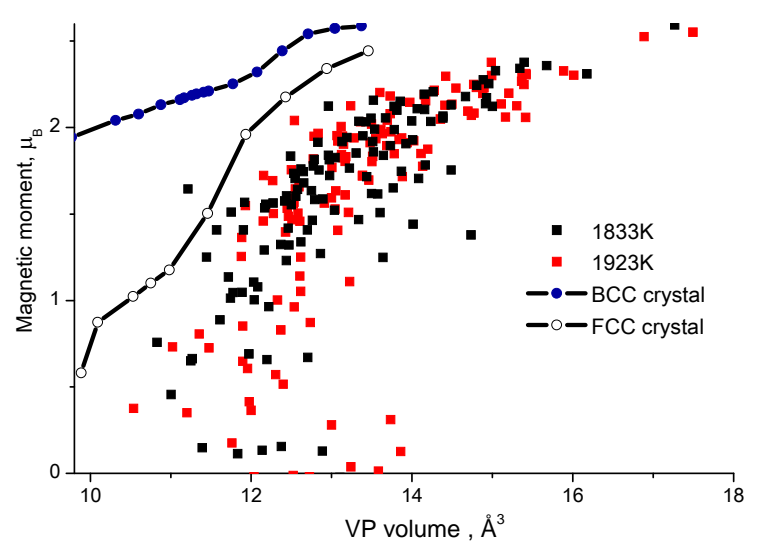

Fig. 1. The distribution of a local magnetic moment on $\mathrm{Fe}$ atom against its Voronoi polyhedral volume. The volume dependence of the magnetic moment of BCC and FCC-phase of crystal iron at $0 \mathrm{~K}$ is shown for comparison.

We observe that volume is not the only factor that determines the magnitude of magnetic moment. This is evident from the spatial fluctuations of magnitudes of magnetic moments of individual atoms, which can be very large, particularly near the Voronoi volume of $12 \AA^{3}$ per atom. In this case the magnetic moment can vary from 0 to nearly $2 \mu_{B}$ even for atoms occupying the same Voronoi volume. To analyze these fluctuations further, we built an autocorrelation function:

$$
f(R)=\frac{1}{4 \pi R^{2}} \sum_{\left|\vec{r}_{i}-\vec{r}_{j}\right|=R} \vec{\mu}_{i}\left(\vec{r}_{i}\right) \cdot \vec{\mu}_{j}\left(\vec{r}_{j}\right)
$$

This function shows us angle distribution between local magnetic moments on atoms at sites $r_{i}$ and $r_{j}$. Positive values of the function correspond to mainly parallel orientation of spins, while negative values correspond to antiparallel orientation. Values close to zero correspond to no correlation between spins orientation. One would expect that such function can describe the short-range magnetic order in the investigated system. The results are plotted in Fig. 2.

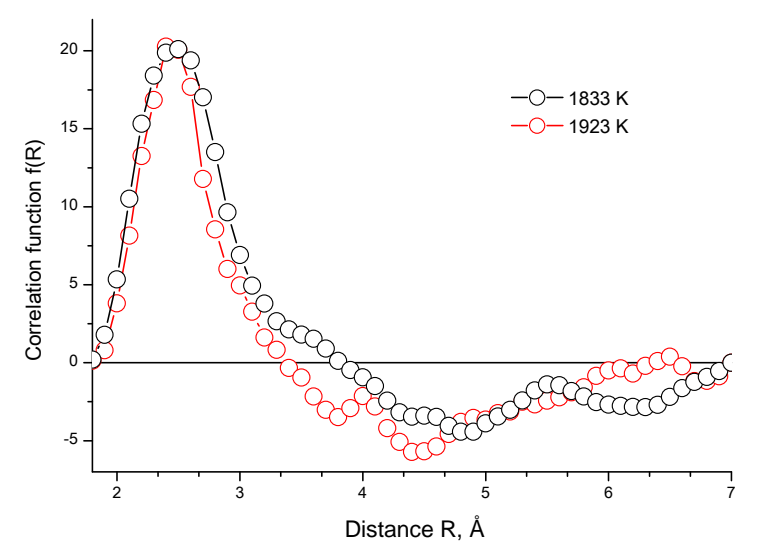

Fig.2. Autocorrelation function $\mathrm{f}(\mathrm{R})$ of liquid iron for two different temperatures. Dot radius corresponds to the standard deviation of the function.
We were able to obtain statistics of at least several thousand atomic pairs for each $R$, thus lowering standard deviation of the function, which is shown in Fig. 2 by dot radii. The results directly indicate existence of small magnetic ordering clusters in liquid iron the volume of which is decreasing with the increase of temperature. The possibility of such state with the strong magnetic correlations is supported by high density of states at the Fermi level which was obtained in our DOS calculations (Fig.3).

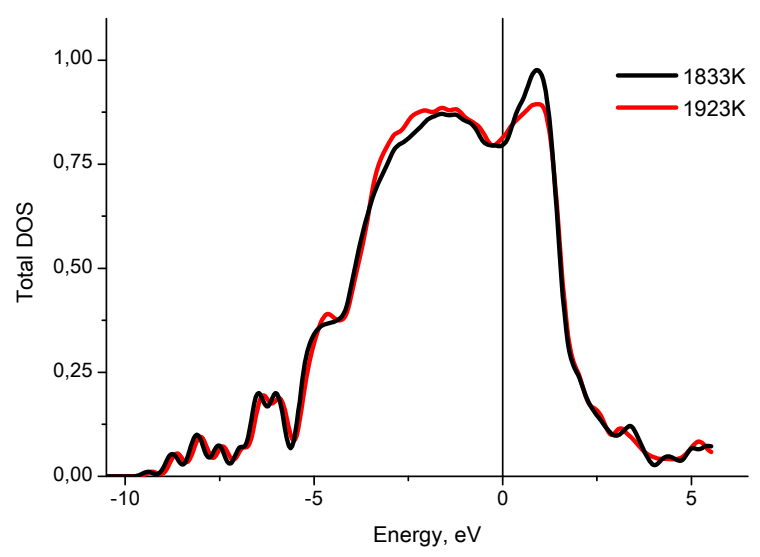

Fig.3. Temperature dependence of density of states (per atom) for liquid iron.

The results of calculations of the temperature dependence of average local magnetic moment for liquid Fe are shown on Fig.4. The magnitude of average moment is close to one for amorphous Fe [16,17], but efficiently lower than the value obtained by the analysis of liquid iron experimental paramagnetic susceptibility data in accordance with Curie - Weiss law[18].

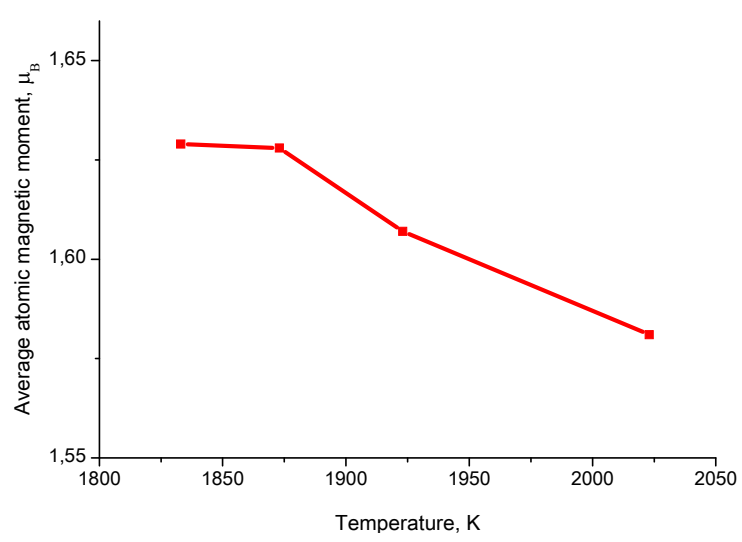

Fig.4. The temperature dependence of average magnetic moment per atom in liquid iron.

To explain the origin of this contradiction, we can turn to Fig. 5. The relaxation of the supercell magnetic moment with time for liquid iron is shown here. The initial state was chosen as relaxed ferromagnetic BCC lattice of Fe. It is clear that at temperature $1923 \mathrm{~K}$ system's ferromagnetic state passes from initial one to the paramagnetic state with magnetisation fluctuations of order $1 / \mathrm{N}^{1 / 2}$, where $\mathrm{N}$ is a number of particles in system. On the contrary at temperature $1833 \mathrm{~K}$ fluctuations becomes larger and this could be 
interpreted as an establishment of a some type of superparamagnetism.

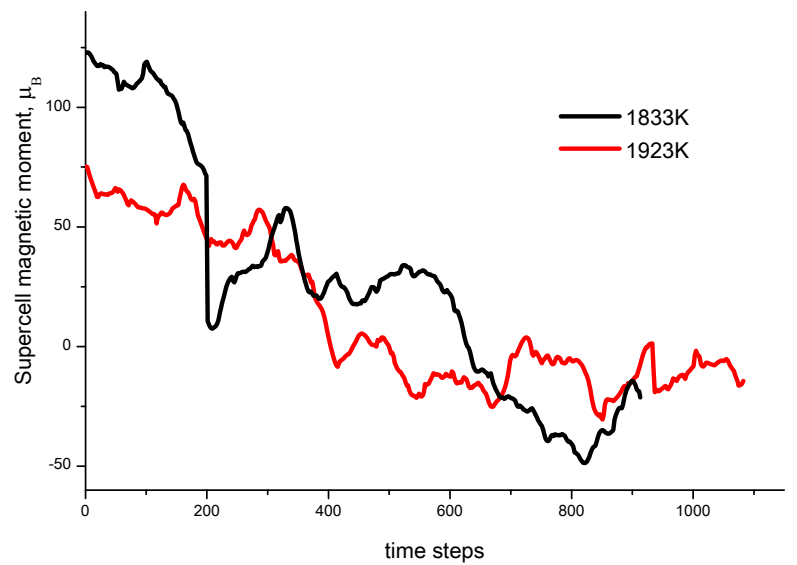

Fig.5. Time evolution of supercell magnetic moment for two different temperatures (initial magnetic state is ferromagnetic BCC-like).

Such superparamagnetic state could be the reason of high magnetic moment value in Curie-Weiss treatment of magnetic susceptibility experiment. This state has high magnetic entropy and gives negative magnetic contribution lowering the total energy value (Fig.6).

At higher temperatures such state becomes unstable and we believe that it could be a reason for liquid-liquid phase transition. We also believe that the transition between superparamagnetic and paramagnetic states in $\mathrm{Fe}$ melts could be accompanied by structural transition and could be reflected in structurally-sensitive properties. You could see two of those properties on Fig.7, such as calculated in present work sphericity coefficient of Voronoi polyhedra or experimental viscosity data[19].

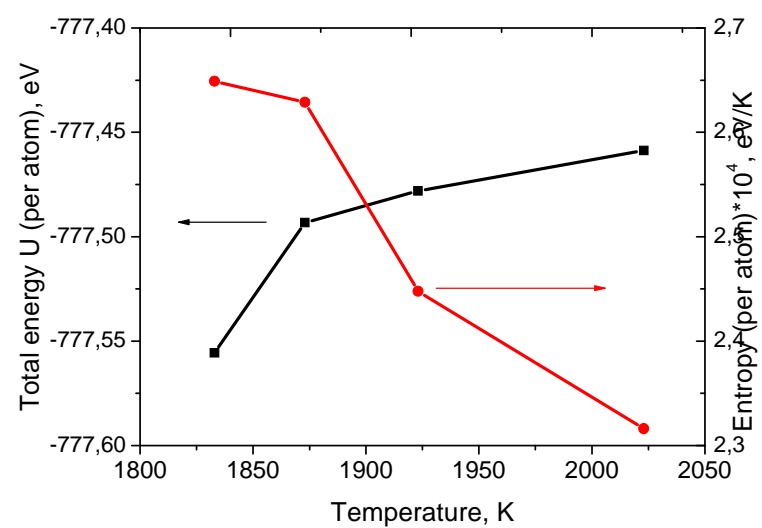

Fig.6. The results of total energy and entropy calculations of liquid $\mathrm{Fe}$ systems as function of temperature.

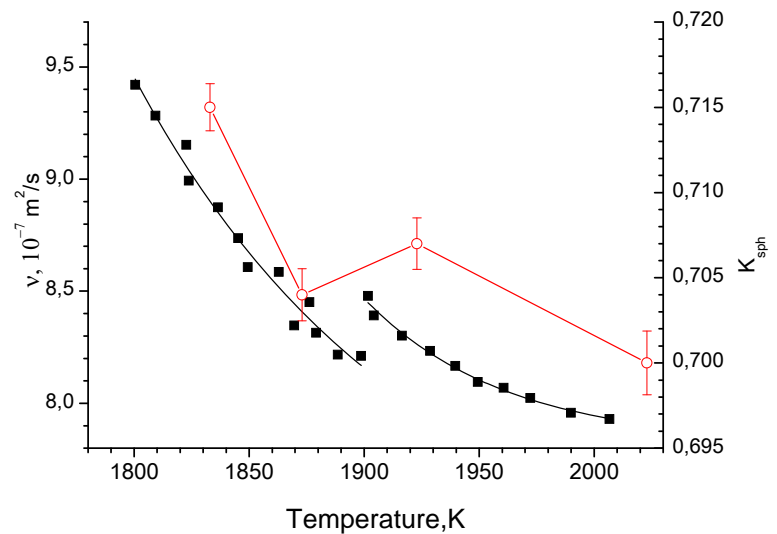

Fig.7. Temperature dependence of the sphericity coefficient of calculated Fe melts structure models (black square) and viscosity experimental data [19] (open circle).

\section{Conclusions}

We might see the existence of the state with short-range magnetic order in liquid iron at temperatures close to the melting point (up to $1923 \mathrm{~K}$ ).

Such superparamagnetic type state has higher magnetic entropy and gives negative magnetic contribution lowering the total energy value. At higher temperatures the state becomes unstable and we believe that it could be a reason for liquid-liquid phase transition.

\section{Acknowledgements}

This work was supported by RFBR grant 09-03-00584-a.

\section{References}

1. Y.Katayama et al. Nature., 403,170(2000)

2. Donavan, E. P. et al., J. Appl. Phys., , 57, 179 (1985)

3. Tanaka, H., Phys. Rev. E, , 62, 6968 (2000)

4. Vertman A.A., Samarin A.M. Properties of iron melts (1969), In Russian

5. Baum B.A. Metallic liquids (1989), in Russian

6. E. M. Haines, V. Heine, and A. Ziegler, J. Phys. F 15, 661(1985)

7. E. M. Haines, R. Clauberg, and R. Feder, Phys. Rev. Lett. 54, 932 (1985)

8. V. Korenman,J. L.Murray, and R. E. Prange, Phys. Rev. B16, 4032 (1977)

9. R. E. Prange and V. Korenman, Phys.Rev. B 19, 4691 (1979)

10. V. Heine and R. Joynt, Europhys. Lett. 5, 81 (1988)

11. J. Soler et al, J. Phys: Condens. Matter, 14, 2745 (2002)

12. Izquierdo et al. PRB, 61, 13639 (2000)

13. J. P. Perdew, K. Burke, and M. Ernzerhof, Phys. Rev. Lett. 77, 3865 (1996).

14. S. G. Louie, S. Froyen, and M. L. Cohen, Phys. Rev. B 26, 1738 (1982). 
15. E. G. Moroni, G. Kresse, J. Hafner, and J.Furthmuller, Phys.Rev.B56,15629,(1997).

16. W.Pui-ai Ma, W. C. Liu, C. H. Wooa and S. L. Dudarev, J. of Appl. Physics 101, 073908 (2007).

17. M.W.Grinstaff, M.B.Salomon K.S.Sulick Phys. Rev. B,(1993),48, 269-273.

18. S.P.Dovgopol, I.A.Zaborovskaya Magnetism and electronic structure of liquid 3d metals (1982) in Russian

19. Novohatskii I. Arkharov V., Kisun'ko V., Doklady of USSR AC (1973). 